Revista de Negócios_ISSN 1980.4431_vol. 19, n. 4, p. 64_83 2014_DOI:10.7867/19804431.2014v19n4p64_83

\title{
IDENTIFICAÇÃO DOS RISCOS EM CADEIAS DE SUPRIMENTOS: um estudo introdutório com empresas da região Sul do Brasil.
}

\section{IDENTIFICATION OF THE RISKS IN SUPPLIES CHAINS: an introductory study with enterprises of the South Region in Brazil.}

\author{
Edson Cezar Aguiar \\ Pontifícia Universidade Católica do Paraná - Brasil \\ edson_cezar@uol.com.br \\ Ubiratã Tortato \\ Pontifícia Universidade Católica do Paraná - Brasil \\ ubirata.tortato@pucpr.br
}

Marilson Alves Gonçalves

(in memoriam)

Recebido em 22 de abril de 2012. Alterado em 13 de agosto de 2014. Aprovado em 09 de outubro de 2014. Editores Responsáveis: Edson Roberto Scharf, Dr. e Marianne Hoeltgebaum, Dr.

Processo de avaliação por double blind review

\section{Resumo}

Com o objetivo de identificar riscos em cadeias de suprimentos e de colocar o tema em evidência esse trabalho inserido nas abordagens teóricas sobre Processos de Negócios, Gerenciamento das Cadeias de Suprimentos e Riscos se concentrou na identificação dos riscos que facilite às empresas participantes de uma cadeia de suprimentos a decisão das ações que devem ser tomadas em relação a eles. A pesquisa é aplicada, exploratória, quantitativa, porém não probabilística e se utilizou de uma survey para a coleta dos dados. Os resultados apontam que os riscos relativos à estrutura organizacional e ambiente externo são os mais representativos para as empresas participantes da pesquisa.

Palavras - chave: riscos, gerenciamento de riscos em cadeias de suprimentos, tipologia dos riscos.

\begin{abstract}
In order to identify risks in supply chains and to give prominence to the subject, the present paper, embedded in the theoretical approaches regarding Business Processes, Supply Chain Management and Risks, had concentrated on the identification of the risks that facilities to the participating enterprises of a supplies chains the decisions of the actions that must be taken in relation to them. The research is applied, exploratory, quantitative, but not probabilistic and has utilized of a survey for the collection of the data. The results point that the risks related to the organizational structure and external environment are the most representative for the participating enterprises on the research.
\end{abstract}

Keywords: risks, supply chain risk management, risk typology. 


\section{Introdução}

A necessidade de enfrentar a competição global fez com que as empresas, além dos esforços para alcançar a eficiência e a atualização tecnológica organizacional, buscassem a evolução de seus processos de gestão de uma realidade individual para o conceito de integração entre as empresas, por meio de organizações virtuais (FLEURY; FLEURY, 2000; PIRES, 2004), conhecidas no meio acadêmico e empresarial como Gerenciamento das Cadeias de Suprimentos (SCM), ou de forma mais simples, Cadeias de Suprimentos (CS).

A CS pode ser considerada como uma forma organizacional de grande potencial de geração de valor e diferenciação para as empresas, constituindo-se, deste modo, como um novo modelo gerencial (PARRA; PIRES, 2003) e introduz uma importante mudança no modelo competitivo, ao considerar que cada vez mais a competição se estabelece, agora, entre as cadeias de suprimento (PIRES, 2004).

Contudo, essa forma organizacional também está exposta a ameaças e riscos, algumas vezes tão severos, que ocasionam a interrupção da produção. Diversos são os exemplos. Em março de 2001, a fábrica da Philips em Albuquerque, no Novo México, foi destruída por um incêndio. A empresa fornecia chips de radiofrequência (RFCs) para as fabricantes de telefones celulares Nokia e Ericsson. Esse problema de fornecimento de chips pela Phillips gerou um prejuízo para a Ericsson de 1,7 bilhão de dólares naquele ano (UNIVERSIA KNOWLEDGE@WHARTON, 2009). Essas interrupções tiveram origem em acidentes provocados pelo homem ou pela natureza. Outras vezes, os eventos de riscos têm origem na gestão empresarial, mas, também fornecem uma ideia da vulnerabilidade das cadeias de suprimentos. Por exemplo, o encerramento das atividades da Chrysler ocorrido no ano de 2001 na planta de Campo Largo, no Brasil, devido a problemas de demanda. Outro exemplo é o da Land Rover, também em 2001, na Inglaterra, em sua relação de fornecimento com a UPF Thompson. A falência do fabricante de chassis UPF-Thompson provocou impacto grave e imediato sobre seu principal cliente, a Land Rover. A UPF era o único fornecedor de chassis para o modelo mais vendido da Land Rover, a Discovery. Essa cadeia de suprimentos esteve em risco, como resultado das perdas sofridas pela UPF em um empreendimento financeiro internacional que não estava relacionado diretamente à cadeia de suprimentos, mas que foi desastroso. Neste sentido a falta de materiais em consequência de atrasos do fornecedor único se constitui em fonte de risco (CHOPRA; SODHI, 2005; ZSIDISIN et al., 2005).

Esses exemplos, basicamente, de ordem prática e gerencial evidenciam que os riscos permeiam todas as cadeias de suprimentos e que possuem potencial de interromper o faturamento das empresas (ZSIDISIN et al, 2005; WAGNER; BODE, 2008). Isso expõe a necessidade das empresas componentes das cadeias de suprimentos a identificarem seus riscos. Entretanto, as releituras da literatura feitas por Normann e Lindroth (2004), Hallikas et al (2004) e Aguiar (2010) revelam que são raros estudos empíricos sobre riscos em cadeias de suprimentos com visão abrangente de várias empresas. Assim sendo, emerge a importância de melhor entendimento sobre os riscos nas cadeias de suprimentos (FURLANETTO, 2002). A revisão bibliográfica sobre Gestão de Riscos em Cadeias de Suprimentos elaborada por Tomas e Alcântara (2013) confirma o crescente interesse, por pesquisadores acerca das questões relativas aos riscos em cadeias de suprimentos, tais como (SODHI; GAK-SON, TANG, 2012; SINGHAL; AGARWAL; MITTAL, 2012). Outras questões, como a antiga priorização de minimização de custos na 
cadeia de suprimentos, tem agora dado espaço na literatura para uma forte advertência à necessidade de identificação dos riscos e vulnerabilidades (MARBLE; THUN, 2006). Ressalte-se que pesquisas sobre o tema ainda são pouco exploradas no Brasil. $\mathrm{Na}$ investigação sobre riscos em cadeias de suprimentos existe outra lacuna na literatura: é a perspectiva dos profissionais praticantes (JÜTTNER, 2005). Dessa forma, as justificativas desse trabalho decorrem da própria atualidade e importância do tema e por tratar-se de um campo pouco explorado empiricamente.

Este artigo buscou identificar os riscos nas cadeias de suprimentos a partir da percepção dos gestores das CS. Pretende, ainda, entre outras contribuições, colocar em evidência este tema, de forma que outros pesquisadores sintam-se estimulados a debaterem sobre riscos, vulnerabilidades, fatores de riscos e gerenciamentos de riscos nas CS, uma vez que não há consenso na academia a respeito de modelos e abordagens, nesta área promissora e vital de investigação.

O presente trabalho está inserido nas abordagens teóricas sobre Processos de Negócios, Modelos de Gestão, Gerenciamento das Cadeias de Suprimentos e Riscos e, concentra-se na identificação dos riscos nas cadeias de suprimentos. Neste sentido, pode ser classificado como uma pesquisa exploratória. Para $o$ alcance do objetivo, utilizou-se de uma revisão de literatura e do instrumento de pesquisa desenvolvido por Aguiar (2010) para a identificação dos riscos das cadeias de suprimentos através de uma survey. Em temos práticos, a contribuição do trabalho estará em responder a seguinte pergunta: quais os riscos existentes nas cadeias de suprimentos?

A estrutura do trabalho compõese de seis partes. Além dessa Introdução, há dois tópicos que tratam especificamente do referencial teórico que dá a sustentabilidade ao artigo: cadeias de suprimentos; gestão dos riscos e identifi- cação dos riscos. Os procedimentos metodológicos, os resultados e a análise dos dados, juntamente, com as considerações finais perfazem os três últimos tópicos.

\section{Cadeia de Suprimentos}

Tradicionalmente, as empresas entendem que são agentes econômicos que existem de forma independente e que precisam competir com outras empresas para sobreviver. Entretanto, esse pensamento, pode ser autodestrutivo caso ele incentive a competição em vez da cooperação (PIRES, 2004). No contexto contemporâneo, em função da impossibilidade de uma única empresa exercer controle sobre o fluxo produtivo, desde a matéria - prima até o consumidor final, elas buscam por relacionamentos com outras empresas (BALLOU, 2006). Entretanto, é necessário que tais relacionamentos sejam pautados pela cooperação e não pela competição ou por relacionamentos conflituosos, para que todas as empresas pertencentes a cadeia de suprimentos possam alcançar vantagens competitivas e muitos dos benefícios da tradicional integração vertical, sem as comuns desvantagens em termos de custo e perda de flexibilidade a ela inerentes (PIRES, 2004).Assim, as cadeias de suprimentos tornaram-se fonte de competitividade para empresas e indústrias. (SOUZA et al., 2012).

O termo que foi proposto pela primeira vez na literatura na década de 80 , ultimamente, tem feito parte do vocabulário diário de executivos, acadêmicos e de público mais amplo. Uma das condições básicas para a concretização e operacionalização de uma cadeia de suprimentos é o efetivo relacionamento colaborativo, que se alicerça em teorias, tais como, Dependência de Recursos, Economia dos Custos de Transação e Administração Estratégica (DI SÉRIO; SAMPAIO, 2001; FURLANETTO, 2002).

Diversas são as definições. É definida como sendo o conjunto de ativida- 
IDENTIFICAÇÃO DOS RISCOS EM CADEIAS DE SUPRIMENTOS: um estudo introdutório com empresas da região Sul do Brasil.

des que envolvem a distribuição do produto para o consumidor final, desde a aquisição de matéria prima, manufatura e também o sistema de informações envolvido (LUMMUS e VOKURKA, 1999). Para Furlaneto (2002) a formação da cadeia de suprimento deve ser uma decisão estratégica, por ser composta de uma rede de empresas. Pode ser entendida como um corpo de conhecimentos que tem a finalidade de coordenar as ações e atividades das diversas empresas que constituem os elos interligados para a produção de bens e serviços a seus consumidores (POIRIER; REITER, 1997). A cadeia de suprimentos é uma rede de empresas, efetivamente responsáveis pela obtenção, produção e distribuição de um determinado produto ou serviço ao cliente final (PIRES, 2004).

Wood Jr. e Zuffo (1998) descrevem o processo evolucionário do conceito, destacando, a importância do melhor gerenciamento dos sistemas complexos inter-relacionados como: planejamento de materiais, gerenciamento de inventários, planejamento de capacidades, sistemas de produção e logística. Segundo Franceschini e Gurgel (2002) o conceito apresentado na Figura 1, trata da integração dos processos de negócios com os componentes de gestão e a estrutura da cadeia de suprimentos. Para esses autores, os processos de negócios são representados por um conjunto de atividades empresariais que agregam valor ao produto ou serviço, segundo a percepção do cliente. $\mathrm{Na}$ visão de Fleury (2000) os processos de negócios de uma CS são os seguintes: relacionamento com os clientes, serviço aos clientes, administração da demanda, atendimento de pedidos, administração do fluxo de produção, suprimentos e desenvolvimento de novos produtos.

Figura 1 - Gerenciamento da Cadeia de Suprimentos

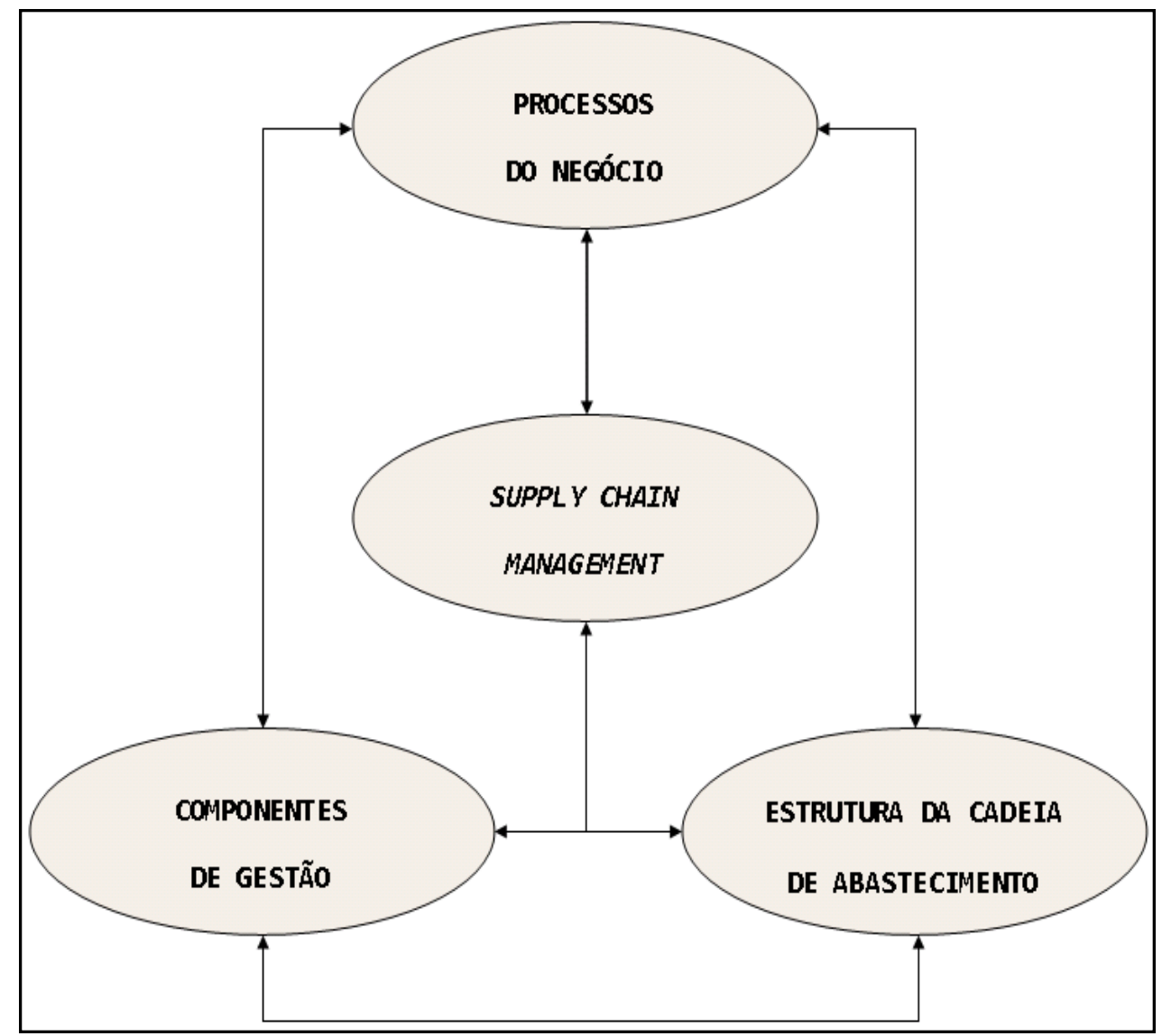

Fonte: Franceschini; Gurgel, 2002. 
Os componentes de gestão, segundo Franceschini e Gurgel (2002), são definidos como o conjunto de atividades empresariais que são necessárias ao planejamento, execução e controle dos processos do negócio. Entre diversas citamse gestão de estoques, distribuição, compras e suprimentos, finanças, previsão de demanda, sistemas de informação, atribuições e direitos de decisão (SILVA FILHO; ALVAREZ; PROENÇA, 2002). Os componentes de gestão devem buscar minimizar os riscos naquelas atividades com mais capacidade de geração de valor do ponto de vista dos investidores (HAHN E KUHN, 2012).

$\mathrm{Na}$ estrutura das CS, a literatura propõe que haja uma organização hierárquica, com papéis bem definidos. Os fornecedores devem estar organizados em níveis, estabelecendo relações cooperativas - com fornecedores no mesmo nível e com fornecedores nos demais níveis e são coordenados (em parte pelo menos) pelos fornecedores nos níveis superiores (mais próximos dos clientes ou mais importantes, na definição dos negócios na cadeia) (LAMBERT; COOPER; PAGH, 1998). Podem-se incluir aspectos relacionados como os papéis das empresas ou unidades produtivas estão distribuídos nas cadeias ou, em outras palavras, como o trabalho está dividido entre os elos da cadeia de suprimentos, e como atividades e processos são realizados.

Atualmente a complexidade das cadeias de suprimentos, com a entrada de maior número de fornecedores e a importância do item para o produto do comprador conduz ao aumento das situações de risco (VILKO E HALLIKAS, 2013 .(Também o aumento das distâncias e rapidez das entregas, as cadeias ficaram mais suscetíveis a rupturas, crescendo de importância a gestão do risco (TANG E MUSA, 2011).

\section{Gestão dos riscos}

O risco é inerente a qualquer atividade na vida pessoal, profissional ou organizacional, entretanto, ainda não foi estabelecida uma definição universalmente reconhecida (WAGNER; BODE, 2008).

$\mathrm{Na}$ área de gestão de empresas, algumas publicações trataram da questão de como definir o risco. Segundo Moore (1983) há duas abordagens distintas: (1) $\mathrm{o}$ risco sendo tanto um perigo quanto uma oportunidade e (2) o risco sendo apenas um perigo A primeira abordagem está de acordo com a prática comum de diversas áreas de pesquisa de negócios, como finanças, por exemplo. Nesta área do conhecimento, as flutuações em torno do valor esperado (média) de uma medida de desempenho são usadas como substituto para o risco, onde o último é igualado à variância e abarca tanto a possibilidade "negativa" quanto a "positiva". Seguindo tais considerações e em comparação à definição geral de March e Shapira (1987, p. 1404) e Jüttner et al. (2003, p. 200) risco é a "variação na distribuição de possíveis resultados, suas possibilidades e seus valores subjetivos".

Por outro lado, para outros autores o risco é visto somente como a possibilidade de prejuízos, danos ou perdas. De acordo com Bastias (1997), risco é uma ou mais condições de uma variável que possuem o potencial suficiente para degradar um sistema, interrompendo e/ ou ocasionando o desvio das metas, de maneira total ou parcial.

Para Ghadge; Dani; Kalawsky (2012) risco é a exposição a sérias perturbações que afetam a capacidade das cadeias de suprimentos de entregar produtos e serviços, bem como, outros requisitos do cliente. Fatores relacionados ao fluxo de material entre os nós pode significativamente reduzir a resiliência 
IDENTIFICAÇÃO DOS RISCOS EM CADEIAS DE SUPRIMENTOS: um estudo introdutório com empresas da região Sul do Brasil.

das cadeias de suprimentos (SVENSSON, 2003).

Esses fatores incluem o número de nós na cadeia de suprimentos, a presença de regulamentos e questões de segurança e congestionamento de portos e restrições da capacidade dos navios. Nesse sentido constitui um risco (BLACKHURST; DUNN; CRAIGHEAD, 2011).

Jackson e Carter (1992) concordam com o fato de que o conceito de risco está associado com a falha de um sistema, sendo a possibilidade de falha usualmente entendida em termos de probabilidades e do impacto.

A noção de que o risco traz essencialmente consequências negativas corresponde à percepção humana comum. March e Shapira (1987), por exemplo, examinaram empiricamente como os gestores detectam os riscos e reagem a eles. Eles descobriram que a maioria tende a exagerar a possibilidade "negativa" do risco. Diversos estudiosos das áreas de gestão de cadeias de suprimentos e gestão do fornecimento adotaram tal visão. Harland; Brenchley; Walker (2003, p.52), por exemplo, discutiram algumas definições e concluíram que o risco na cadeia de suprimentos está associado à "possibilidade de perigo, dano, perda, prejuízo ou qualquer outra consequência indesejada". Como exemplo, cita-se o caso da Petrobrás na Bolívia: em maio de 2006, o recémeleito Presidente da Bolívia, Evo Morales, anunciou a nacionalização da exploração de petróleo e gás naquele país. Essa medida afetou diretamente empresas como Petrobras (do Brasil Repsol YPF
(Espanha e Argentina), British Gas e British Petroleum (Reino Unido), Total (França), Dong Wong (Coréia) e Canadian Energy (Canadá). Somente a Petrobras já havia investido na Bolívia, desde 1996, cerca de US\$ 1,5 bilhão, além de US\$ 2 bilhões no transporte do gás da Bolívia para o Brasil (FOLHA DE SÃO PAULO, 01/05/2006).

Considerando o impacto de interrupções recentes em cadeias de suprimentos, como o tsunami no Japão no ano de 2011 quando várias cadeias de suprimentos tiveram seus fluxos produtivos interrompidos, cremos que a última noção de risco, como sendo unicamente negativo, é a que melhor corresponde à realidade dos negócios das cadeias de suprimentos. Portanto, o risco é igualado às consequências prejudiciais de uma interrupção na cadeia de suprimentos - a perda ou dano que se teve. Para Nutini (2005), nos cenários estratégicos complexos da atualidade a Gestão de Risco é um componente essencial da gestão para a excelência. Depreende-se que as organizações precisam assumir e gerenciar riscos para gerar valor a seus acionistas (shareholders) ou interessados (stakeholders). O gerenciamento de riscos baseia-se na identificação, análise, priorização, avaliação, monitoração e resultados do desempenho (Figura 2), com o objetivo de detectar riscos e implantação de ações para reduzir a probabilidade de ocorrências e/ou minimizar o impacto negativo das mesmas sobre os resultados (AGUIAR, 2010).

Figura 2 - Fases da Gerência de Riscos

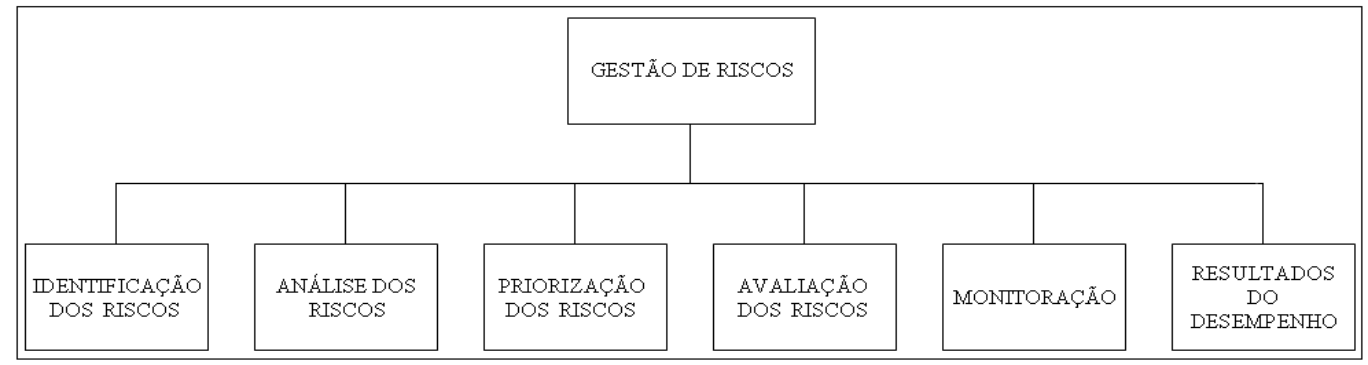

Fonte: Aguiar, 2010. 
O interesse de estudo desse trabalho é a identificação dos riscos devido a relevância que essa fase da gestão de riscos em cadeias de suprimentos obteve a partir de eventos que causaram interrupções ou desvios nas cadeias de suprimentos. Segundo Shi (2004) para a identificação eficaz de riscos em cadeia de suprimentos, é proveitoso usar uma taxonomia para classificar os riscos. Essa taxonomia pode ser obtida através de brainstorming que, revela tendências - chave de ordem econômica, tecnológica e cultural e pode ser utilizada para enumerar um conjunto de fatores de riscos já existentes e futuros que podem afetar o desempenho das empresas inseridas em cadeias de suprimentos. Ainda segundo o autor, outra maneira é a análise da situação, particularmente eficaz na identificação de riscos resultantes de novas tecnologias, mudanças na estrutura e dinâmica do setor de negócios. O mapeamento de processos também pode ser usado para criar uma taxonomia de riscos e é particularmente proveitoso para identificar os riscos associados a execuções insatisfatórias. A análise histórica é outra maneira de identificar e examinar riscos já existentes e suas interações com os fatores de riscos que estão surgindo para as empresas das cadeias de suprimentos. Uma última forma, segundo (Shi,2004), porém não menos importante, para a obtenção de riscos em cadeias de suprimentos é a revisão de literatura.

A "origem dos riscos" (Figura 3) refere-se ao tipo de ambiente, externo ou interno, onde o fator de risco se localiza. Os riscos externos são ocorrências associadas ao ambiente macroeconômico, político, social, natural ou setorial em que a cadeia de suprimentos opera. São exemplos: tecnologias emergentes, ações da concorrência, mudança no cenário político, conflitos sociais, demanda, entre outros. A organização, em geral, não consegue intervir diretamente sobre estes eventos e terá, portanto, uma ação predominantemente reativa. Isto não significa que os riscos externos não possam ser "gerenciados"; pelo contrário, é fundamental que a organização esteja bem preparada para essa ação reativa. Os riscos internos são eventos originados na própria estrutura da organização, pelos seus processos, seu quadro de pessoal ou de seu ambiente de tecnologia.

Figura 3 - Origem dos Riscos

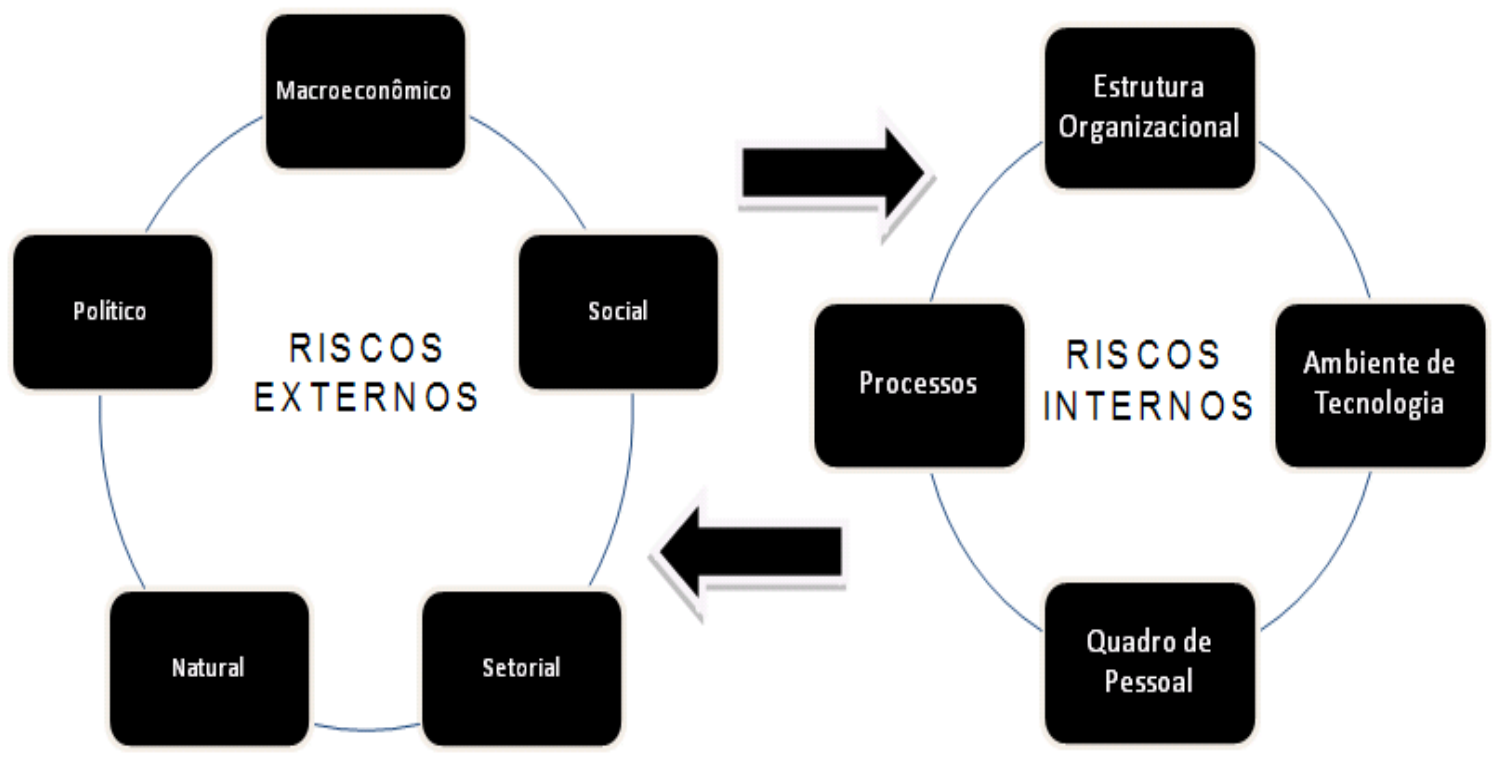

Fonte: Os Autores 
A fase de identificação dos riscos potenciais ao negócio propõe que sejam percebidas todas as consequências adversas que sejam prováveis de ocorrerem nas cadeias de suprimentos (HULETT, 2002).

\section{Procedimentos metodológicos}

Neste trabalho, se seguiu a noção do risco como o desvio negativo do valor esperado de determinada medida de desempenho ou de interrupções que resultaram em consequências negativas para as empresas e cadeias de suprimentos. Assim, a linha de raciocínio adotada neste artigo é a de que o risco é caracterizado como um fator constituinte e condicionante de um sistema industrial, intrínseco a todas as atividades desenvolvidas pelas empresas e cadeia de suprimentos, que deve ser tratado com a devida importância. Isso torna imprescindível a identificação dos riscos aos quais estão expostas. Para Alberton (1996) dirigir, estrategicamente, os riscos, supõe que eles serão considerados como parte da competitividade empresarial. Dessa forma, a população alvo da pesquisa é o conjunto de profissionais de logística e cadeias de suprimentos (gerentes e analistas seniores) das empresas filiadas às federações das indústrias dos três estados do sul do Brasil, por conta de seu conhecimento sobre práticas de gestão das cadeias de suprimentos e logística.

A pesquisa quanto à natureza é aplicada e quanto à abordagem do problema, ela é quantitativa, porém nãoprobabilística. Também é uma pesquisa do tipo corte-transversal, isto é, os dados foram coletados em um dado momento no tempo, entre 15 de janeiro a 15 de março de 2010. Quanto aos objetivos a pesquisa é exploratória. A meta de uma pesquisa do tipo exploratória é satisfazer à curiosidade do pesquisador, fornecer uma melhor compreensão do tema e identificar sua relevância (GIL, 2002). Quanto aos procedimentos técnicos utili- zados para atender aos objetivos da pesquisa, combinaram-se métodos e técnicas como entrevistas, survey e análise estatística de dados. A pesquisa survey é definida como a maneira de coletar dados ou informações sobre particularidades, ações ou opiniões de um determinado grupo de pessoas, representantes de uma determinada população-alvo, por meio do instrumento de pesquisa (questionário). A análise estatística dos dados se utilizou de duas ferramentas estatísticas: o coeficiente de variação e a média aritmética, conforme usadas por Wagner e Bode (2008).

\subsection{Desenvolvimento do Questionário e Coleta de Dados}

$\mathrm{O}$ instrumento de pesquisa é adaptado do trabalho de Aguiar (2010) no que se refere aos riscos em cadeias de suprimentos e de revisão da literatura que determinou um agrupamento inicial de itens. Esse agrupamento inicial de itens foi revisado, junto a alguns pesquisadores e executivos de gestão de cadeias de suprimentos. Após a revisão, alguns itens foram eliminados ou reformulados. Dos cinquenta e um itens iniciais, quarenta e nove itens remanescentes foram incorporados em um questionário e pré-testados.

O questionário usado no estudo era composto de cinco blocos. O primeiro bloco era referente ao construto processos de negócios das cadeias de suprimentos, composto por dois conceitos: relacionamentos com clientes, composto por 13 itens e fornecedores e administração da demanda, composto por seis itens.

$\mathrm{O}$ segundo bloco se referia ao construto estrutura organizacional, composto por três itens. O terceiro bloco se referia aos componentes de gestão e era composto por dois conceitos, cada um deles com um item. O quarto e quinto bloco se referiam ao construto ambiente externo e ambiente interno das cadeias de suprimentos. O construto ambiente 
externo era composto por cinco conceitos: aspectos macroeconômicos, composto por dois itens; legislação, também composto por dois itens; aspectos político-sociais, composto por um item; desastres naturais, também compostos por um item; setoriais, composto por um item e de infraestrutura, composto por três itens. O ambiente interno era formado por quatro conceitos: ambiente tecnológico, processos, fluxo de informação e desenvolvimento de competências e pessoas.

Quadro 1 - Tipologia para os Riscos em Cadeias de Suprimentos

\begin{tabular}{|c|c|c|}
\hline $\begin{array}{l}\text { CONSTRUTO } \\
\text { PROCESSOS DE } \\
\text { NEGÓCIOS }\end{array}$ & CONCEITO & VARIÁVEL \\
\hline \multirow{13}{*}{$\begin{array}{l}\text { Relacionamento } \\
\text { com } \\
\text { Clientes e Fornece- } \\
\text { dores }\end{array}$} & $\begin{array}{l}\text { Desalinhamento estratégico (existência de conflito de } \\
\text { objetivos) entre os membros da cadeia de suprimentos. }\end{array}$ & RCF1 \\
\hline & Oportunismo por parte de fornecedores e/ou clientes. & $\mathrm{RCF} 2$ \\
\hline & $\begin{array}{l}\text { Falta de coordenação no relacionamento interorganiza- } \\
\text { cional. }\end{array}$ & RCF3 \\
\hline & $\begin{array}{c}\text { Aumento da dependência entre empresas, devido inves- } \\
\text { timentos específicos, tais como, fábricas, instalações e } \\
\text { máquinas. }\end{array}$ & RCF4 \\
\hline & $\begin{array}{l}\text { Baixa capacidade dos fornecedores em cumprir com as } \\
\text { quantidades programadas. }\end{array}$ & RCF5 \\
\hline & Problemas de qualidade do fornecedor. & RCF6 \\
\hline & Falhas de fornecedores em gerar reduções de custos. & RCF7 \\
\hline & $\begin{array}{c}\text { Desempenho logístico insatisfatório dos provedores de } \\
\text { serviços logísticos. }\end{array}$ & RCF8 \\
\hline & Manter fornecedores em regiões geográficas de risco. & RCF9 \\
\hline & $\begin{array}{l}\text { Falta de capacidade dos fornecedores em cumprir com } \\
\text { os prazos de entrega. }\end{array}$ & RCF10 \\
\hline & $\begin{array}{l}\text { Falta de materiais em consequência de interrupção ou } \\
\text { atrasos de fornecedor único. }\end{array}$ & RCF11 \\
\hline & $\begin{array}{l}\text { Falta dos recursos necessários (poder, financeiro e co- } \\
\text { nhecimento tecnológico) para influenciar fornecedores } \\
\text { e/ou clientes. }\end{array}$ & RCF12 \\
\hline & $\begin{array}{c}\text { Falta de mecanismos para monitorar e informar o princi- } \\
\text { pal sobre as atividades e desempenho do agente }\end{array}$ & RCF13 \\
\hline $\begin{array}{l}\text { Administração da } \\
\text { Demanda }\end{array}$ & Demanda do cliente não prevista ou muito volátil. & DEM1 \\
\hline
\end{tabular}


IDENTIFICAÇÃO DOS RISCOS EM CADEIAS DE SUPRIMENTOS: um estudo introdutório com empresas da região Sul do Brasil.

\begin{tabular}{|c|c|c|}
\hline & $\begin{array}{l}\text { Informações insuficientes ou distorcidas dadas pelos } \\
\text { clientes sobre quantidades de pedidos ou demanda. }\end{array}$ & DEM2 \\
\hline & $\begin{array}{l}\text { Falta de habilidade para lidar com mudança de volume } \\
\text { na demanda. }\end{array}$ & DEM3 \\
\hline & $\begin{array}{l}\text { Falta de habilidade para lidar com mudança de produto } \\
\text { na demanda. }\end{array}$ & DEM4 \\
\hline & $\begin{array}{c}\text { Falha na confiabilidade de entrega (descumprimento das } \\
\text { datas entregas). }\end{array}$ & DEM5 \\
\hline & $\begin{array}{l}\text { Falha na rapidez de entrega (aumento nos tempos entre o } \\
\text { recebimento do pedido e a entrega ao cliente). }\end{array}$ & DEM6 \\
\hline $\begin{array}{l}\text { CONSTRUTO ES- } \\
\text { TRUTURA ORGA- } \\
\text { NIZACIONAL }\end{array}$ & CONCEITO & VARIÁVEL \\
\hline \multirow{3}{*}{$\begin{array}{l}\text { Estrutura Organiza- } \\
\text { cional }\end{array}$} & Dispersão geográfica de fornecedores e clientes. & ESO1 \\
\hline & $\begin{array}{l}\text { Devido à complexidade estrutural (fluxos de informa- } \\
\text { ções, materiais e produtos, número de níveis de forneci- } \\
\text { mento). }\end{array}$ & $\mathrm{ESO} 2$ \\
\hline & $\begin{array}{l}\text { Devido à demora em ajustar a estrutura das empresas e } \\
\text { da cadeia de suprimentos às variações de demanda. }\end{array}$ & ESO 3 \\
\hline $\begin{array}{l}\text { CONSTRUTO } \\
\text { COMPONENTES } \\
\text { DE GESTÃO }\end{array}$ & CONCEITO & VARIÁVEL \\
\hline Gestão de Estoques & $\begin{array}{l}\text { Falta de materiais em consequência da eliminação dos } \\
\text { estoques-pulmão. }\end{array}$ & CGT1 \\
\hline Gestão Financeira & $\begin{array}{l}\text { Inadimplência repentina de um fornecedor (por conta de } \\
\text { falência ou por aquisição por empresas concorrentes). }\end{array}$ & CGT2 \\
\hline $\begin{array}{l}\text { CONSTRUTO AM- } \\
\text { BIENTE EXTER- } \\
\text { NO }\end{array}$ & CONCEITO & VARIÁVEL \\
\hline \multirow[t]{2}{*}{ Macroeconômicos } & Flutuações cambiais. & AEX1 \\
\hline & Globalização da concorrência & AEX2 \\
\hline \multirow[t]{2}{*}{ Legislação } & $\begin{array}{l}\text { Mudanças por conta da introdução de novas leis, regula- } \\
\text { mentações alfandegárias, trabalhistas, etc. }\end{array}$ & AEX3 \\
\hline & $\begin{array}{l}\text { Barreiras ambientais (meio-ambiente) para a operação de } \\
\text { cadeias de suprimentos. }\end{array}$ & AEX4 \\
\hline Políticos Sociais & $\begin{array}{l}\text { Instabilidade política, guerra, agitação civil, greves, rou- } \\
\text { bo de carga, terrorismo ou outras crises sociopolíticas. }\end{array}$ & AEX5 \\
\hline Desastres naturais & $\begin{array}{l}\text { Desastres naturais (terremotos, enchentes, climas extre- } \\
\text { mos, tsunami, por exemplo). }\end{array}$ & AEX6 \\
\hline Setoriais & $\begin{array}{l}\text { Inabilidade de se manter atualizada em relação ao desen- } \\
\text { volvimento tecnológico do setor. }\end{array}$ & AEX7 \\
\hline Infraestrutura & Condições da infraestrutura externa de TI. & AEX8 \\
\hline
\end{tabular}




\begin{tabular}{|c|c|c|}
\hline & $\begin{array}{c}\text { Condições da infraestrutura de transportes (estradas, aero- } \\
\text { portos e portos) }\end{array}$ & AEX9 \\
\hline & Condições de uso do sistema de energia. & AEX10 \\
\hline $\begin{array}{l}\text { CONSTRUTO } \\
\text { AMBIENTE IN- } \\
\text { TERNO }\end{array}$ & CONCEITO & VARIÁVEL \\
\hline \multirow[t]{4}{*}{$\begin{array}{l}\text { Ambiente Tecno- } \\
\text { lógico }\end{array}$} & $\begin{array}{l}\text { Falta de qualidade do projeto e operação do sistema produ- } \\
\text { tivo, tanto pela visão de gestão como tecnológica. }\end{array}$ & AIN1 \\
\hline & Não aceitação pelo mercado dos novos produtos & AIN2 \\
\hline & $\begin{array}{l}\text { Ritmo inferior à média do setor, na geração de inovações } \\
\text { em produtos e processos. }\end{array}$ & AIN3 \\
\hline & $\begin{array}{l}\text { Falta de regras, normas e procedimentos que regem a ma- } \\
\text { neira como uma empresa controla os processos de compra, } \\
\text { produção, qualidade, inovação e de informações. }\end{array}$ & AIN4 \\
\hline Processos & $\begin{array}{c}\text { Falta de eficiência dos processos que permitem o fluxo de } \\
\text { materiais, desde a matéria-prima até o produto final (ou } \\
\text { usuário final). }\end{array}$ & AIN5 \\
\hline \multirow{6}{*}{$\begin{array}{l}\text { Desenvolvimento } \\
\text { Competências e } \\
\text { Pessoas }\end{array}$} & $\begin{array}{l}\text { Clara definição de funções e responsabilidades que promo- } \\
\text { va a autonomia e conscientização. }\end{array}$ & AIN6 \\
\hline & Conjunto de habilidades (competências) dos funcionários. & AIN7 \\
\hline & $\begin{array}{l}\text { Existência de processo de gestão de desempenho que defi- } \\
\text { na metas, mensuração e feedback. }\end{array}$ & AIN8 \\
\hline & Desenvolvimento do trabalho em equipes multifuncionais. & AIN9 \\
\hline & $\begin{array}{l}\text { Existência de um processo de desenvolvimento de funcio- } \\
\text { nários através da gestão de aquisição de competências para } \\
\text { alcançar a mobilidade funcional. }\end{array}$ & AIN10 \\
\hline & $\begin{array}{c}\text { Regras, normas e procedimentos que regem a maneira } \\
\text { como uma empresa controla a conduta de seus funcioná- } \\
\text { rios. }\end{array}$ & AIN11 \\
\hline \multirow{4}{*}{$\begin{array}{l}\text { Fluxo de Informa- } \\
\text { ção }\end{array}$} & Condições da infraestrutura interna de TI & AIN12 \\
\hline & Consideração dos fatores de riscos nas decisões. & AIN13 \\
\hline & $\begin{array}{l}\text { Falta de informações atuais e confiáveis devido ao não } \\
\text { compartilhamento de informações. }\end{array}$ & AIN14 \\
\hline & Falta de capacidade da empresa em processar informações. & AIN15 \\
\hline
\end{tabular}

Fonte: Adaptado de AGUIAR, 2010.

Foi solicitado aos respondentes que as respostas deveriam contemplar o período dos últimos três anos e as variáveis do modelo foram operacionalizadas em uma escala de intensidade tipo Likert de 7 pontos, conforme segue: "Por favor indique o quanto sua unidade de negócios foi afetada negativamente nos últimos 3 anos por cada um dos seguintes riscos em cadeias de suprimentos" (1: nem um pouco - 7: fortemente)". Com base nesse questionário, os dados foram coletados gerando uma taxa de resposta relativamente alta, de cerca 9,8\% 
IDENTIFICAÇÃO DOS RISCOS EM CADEIAS DE SUPRIMENTOS: um estudo introdutório com empresas da região Sul do Brasil.

(234 respostas de 2381 questionários enviados) considerando o tempo restrito dos executivos (TOMASKOVIC-DEVEY et al., 1994). O número de respostas obtidas sugere a representatividade de diversos níveis das cadeias de suprimentos.

\subsection{Caracterização da Amostra}

A amostra contemplou empresas industriais. $\mathrm{O}$ número de funcionários variou entre 20 até 1000 funcionários, o número de empresas por setor variou entre 39 até 95 empresas, proporcionando, portanto, uma amostra heterogênea. Dada a gama das empresas estudadas, seus tamanhos e a diversidades dos setores, não houve uma razão evidente para que se esperasse qualquer viés sistemático nos resultados. A Tabela 1 apresenta o setor de atuação da empresa.

Tabela 1 - Empresas por Setor de Atuação

\begin{tabular}{|c|c|c|}
\hline Setor de Atuação & $\mathbf{N}^{\circ}$ de empresas & $\mathbf{N}^{\circ}$ de empresas (\%) \\
\hline $\begin{array}{c}\text { Eletroeletrônica e fabricantes de } \\
\text { produtos de informática }\end{array}$ & 95 & $40,6 \%$ \\
\hline Mecânica & 61 & $26,0 \%$ \\
\hline Têxtil & 39 & $16,7 \%$ \\
\hline Madeira e Móveis & 39 & $16,7 \%$ \\
\hline Total & 234 & $100,00 \%$ \\
\hline
\end{tabular}

Fonte: Adaptado de AGUIAR, 2010.

Não se focou em um setor específico da indústria porque se tentou obter uma ideia mais geral dos riscos em cadeias de suprimentos na região sul do Brasil.

Quanto à área de atuação dos respondentes, conforme Tabela 2, a maioria

dos deles ocupa cargos administrativos em logística e gestão da cadeia de suprimentos $(33,3 \%)$, na área de compras $(32,48 \%)$ ou na área de produção $(30,77 \%)$.

Tabela 2 - Área de Atuação do Respondente

\begin{tabular}{|c|c|c|}
\hline Área de Atuação do respondente & $\mathbf{N}^{\circ}$ de empresas & $\mathbf{N}^{\circ}$ de empresas (\%) \\
\hline Logística/gestão da cadeia de suprimentos & 78 & $33,3 \%$ \\
\hline Compras & 76 & $32,5 \%$ \\
\hline Produção & 72 & $30,8 \%$ \\
\hline Diretor ou proprietário & 3 & $1,3 \%$ \\
\hline Contabilidade & 3 & $1,3 \%$ \\
\hline Vendas & 1 & $0,4 \%$ \\
\hline Outros cargos & 1 & $0,4 \%$ \\
\hline Total geral & 234 & $100,00 \%$ \\
\hline
\end{tabular}

Fonte: Adaptado de AGUIAR, 2010. 


\section{Resultado e Análise de dados}

Com base na amostra obtida de 234 executivos atuando nas áreas de produção, logística e gestão de cadeias de suprimentos, o Quadro 1 apresenta os resultados do estudo fornecendo um panorama da importância de determinados riscos na cadeia de suprimentos da região Sul do Brasil.

Quadro 2 - Médias e Desvios Padrão dos Riscos em Cadeias de Suprimentos e Sua Preponderância

\begin{tabular}{|c|c|c|c|}
\hline VARIÁVEL & MÉDIA & $\begin{array}{l}\text { DESVIO } \\
\text { PADRÃO }\end{array}$ & $\begin{array}{l}\text { COEFICIENTE DE } \\
\text { VARIAÇÃO }\end{array}$ \\
\hline $\begin{array}{l}\text { RISCO RELATIVO AO } \\
\text { AMBIENTE EXTER- } \\
\text { NO }\end{array}$ & 5.01 & & \\
\hline AEX1 & 2,36 & 1,08 & 45,62 \\
\hline AEX2 & 4,85 & 0,75 & 15,56 \\
\hline AEX3 & 1,47 & 0,92 & 62,58 \\
\hline AEX4 & 2,99 & 0,66 & 22,25 \\
\hline AEX5 & 4,08 & 0,67 & 16,44 \\
\hline AEX6 & 6,55 & 1,16 & 17,67 \\
\hline AEX7 & 5,85 & 0,81 & 13,84 \\
\hline AEX8 & 6,67 & 0,80 & 12,04 \\
\hline AEX9 & 3,32 & 0,97 & 30,31 \\
\hline AEX10 & 4,06 & 0,67 & 16,44 \\
\hline $\begin{array}{l}\text { RISCO RELATIVO AO } \\
\text { AMBIENTE INTERNO }\end{array}$ & 4.02 & & \\
\hline AIN1 & 1,65 & 1,16 & 70,28 \\
\hline AIN2 & 3,12 & 0,89 & 28,38 \\
\hline AIN3 & 5,86 & 0,78 & 13,30 \\
\hline AIN4 & 2,36 & 1,14 & 48,18 \\
\hline AIN5 & 4,10 & 0,78 & 19,10 \\
\hline AIN6 & 3,07 & 0,58 & 18,97 \\
\hline AIN7 & 4,90 & 0,89 & 18,22 \\
\hline AIN8 & 2,13 & 0,72 & 33,74 \\
\hline AIN9 & 3,06 & 0,72 & 23,51 \\
\hline AIN10 & 4,13 & 0,93 & 22,56 \\
\hline AIN11 & 1,89 & 1,27 & 66,95 \\
\hline AIN12 & 4,80 & 0,79 & 16,42 \\
\hline AIN13 & 2,16 & 0,73 & 33,69 \\
\hline AIN14 & 4,11 & 0,73 & 17,82 \\
\hline AIN15 & 4,86 & 0,72 & 14,86 \\
\hline
\end{tabular}


IDENTIFICAÇÃO DOS RISCOS EM CADEIAS DE SUPRIMENTOS: um estudo introdutório com empresas da região Sul do Brasil.

\begin{tabular}{|c|c|c|c|}
\hline $\begin{array}{c}\text { RISCOS RELATIVOS } \\
\text { AOS } \\
\text { COMPONENTES DE } \\
\text { GESTÃO }\end{array}$ & 4.34 & & \\
\hline CGT1 & 5,79 & 0,88 & 15,26 \\
\hline CGT2 & 2,88 & 0,54 & 18,85 \\
\hline $\begin{array}{c}\text { RISCOS RELATIVOS } \\
\dot{A} \\
\text { DEMANDA }\end{array}$ & 4.53 & & \\
\hline DEM1 & 4,10 & 0,68 & 16,56 \\
\hline DEM2 & 5,92 & 0,63 & 10,64 \\
\hline DEM3 & 4,11 & 0,71 & 17,35 \\
\hline DEM4 & 4,86 & 0,94 & 19,32 \\
\hline DEM5 & 3,19 & 0,85 & 26,61 \\
\hline DEM6 & 5,02 & 0,67 & 13,36 \\
\hline $\begin{array}{c}\text { RISCOS RELATIVOS } \\
\dot{A} \\
\text { ESTRUTURA ORGA- } \\
\text { NIZACIONAL }\end{array}$ & 6.62 & & \\
\hline ESO1 & 6,56 & 1,05 & 15,98 \\
\hline ESO2 & 6,62 & 0,88 & 13,25 \\
\hline ESO3 & 6,69 & 0,78 & 11,66 \\
\hline $\begin{array}{c}\text { RISCOS DOS RELA- } \\
\text { CIONAMENTOS CL- } \\
\text { IENTE/ } \\
\text { FORNECEDORES }\end{array}$ & 4.84 & & \\
\hline RCF1 & 6,58 & 0,91 & 13,90 \\
\hline RCF2 & 5,74 & 0,93 & 16,24 \\
\hline RCF3 & 2,17 & 0,74 & 34,01 \\
\hline RCF4 & 4,12 & 0,73 & 17,75 \\
\hline RCF5 & 5,09 & 0,66 & 12,94 \\
\hline RCF6 & 3,17 & 0,77 & 24,37 \\
\hline RCF7 & 5,82 & 0,82 & 14,12 \\
\hline RCF8 & 3,08 & 0,63 & 20,55 \\
\hline RCF9 & 3,85 & 0,84 & 21,91 \\
\hline RCF10 & 6,62 & 0,97 & 14,60 \\
\hline RCF11 & 4,06 & 0,81 & 19,93 \\
\hline RCF12 & 4,18 & 0,91 & 21,65 \\
\hline RCF13 & 5,82 & 0,83 & 14,22 \\
\hline
\end{tabular}

Fonte: Adaptado de AGUIAR, 2010. 
O Quadro 2 apresenta os riscos em cadeias de suprimentos que foram investigados, suas médias, desvios padrão (DP) e coeficientes de variação de Pearson $(\mathrm{CV})$.

Foram excluídos aqueles com coeficiente de variação $(\mathrm{CV})>30 \%$, porque se o CV é maior do que $30 \%$, a distribuição é dita heterogênea, e os dados estão muito dispersos em torno da média. A razão para a exclusão das variáveis pelo coeficiente de variação é que as variáveis com $\mathrm{CV}>30 \%$ podem causar instabilidade em parâmetros estatísticos de algumas ferramentas de análise estatística (HAIR JR. et al., 2005). Não é o caso nesta análise, mas assim se preferiu por uma questão de compatibilidade com outras análises que serão feitas a partir desses dados. Foram encontrados 8 CVs com variação maior do que $30 \%$. Assim, foram excluídas 8 medidas e permaneceram 41 delas. Os desvios-padrão demonstram pouca variabilidade em torno da média, indicando um conjunto de respostas relativamente homogêneo. Em relação aos blocos de riscos, a média entre os seis construtos utilizados no trabalho é igual a 4,89. Tomando-se esse valor como base, valores médios dos construtos iguais ou maiores do que esse valor médio representarão riscos que exigem maior atenção. Os números revelam que no que se referem aos construtos dos riscos em cadeias de suprimentos, os riscos relativos às

estruturas organizacionais (média = $6,62)$ e ao ambiente externo $(5,01)$ representam os riscos que exigem atenção nas cadeias de suprimentos estudadas. $\mathrm{O}$ construto relativo a clientes e fornecedores $(4,84)$ tem valor bastante próximo à média, o que também requer atenção dos gestores. Curiosamente, os riscos relativos à demanda (média $=4,53$ ), na opinião dos respondentes não representam problemas para as cadeias de suprimentos estudadas. Pode ser reflexo do bom momento econômico pelo qual passava a economia brasileira. Os riscos relativos aos componentes de gestão $(4,34)$ e os riscos do ambiente interno das cadeias de suprimentos $(4,02)$ situam-se abaixo da média, o que representa que sobre esses dois construtos existe evolução e atendimento aos pressupostos teóricos da gestão de empresas.

Adotando-se para a análise dos itens, o critério utilizado para a análise dos blocos os números revelam que existem vinte e uma medidas com valores maiores do que a média que é igual a 4,71. Mais especificamente, na opinião dos respondentes, afetaram consideravelmente as empresas nos últimos três anos, principalmente, os seguintes riscos: ESO3 - demora em ajustar a estrutura das empresas e da cadeia de suprimentos às variações de demanda; AEX8 - Condições da infraestrutura externa de TI; RCF10 - Falta de capacidade dos fornecedores em cumprir com os prazos de entrega; ESO2 - Devido à complexidade estrutural (fluxos de informações, materiais e produtos, número de níveis de fornecimento); RCF1 - Desalinhamento estratégico (existência de conflito de objetivos) entre os membros da cadeia de suprimentos; ESO1 - Dispersão geográfica de fornecedores e clientes; AEX6 Desastres naturais (terremotos, enchentes, climas extremos, tsunami, por exemplo); DEM2 - Informações insuficientes ou distorcidas dadas pelos clientes sobre quantidades de pedidos ou demanda; AIN3 - Ritmo inferior à média do setor, na geração de inovações em produtos $\mathrm{e}$ processos; AEX7 - Inabilidade de se manter atualizada em relação ao desenvolvimento tecnológico do setor; RCF7 Falhas de fornecedores em gerar reduções de custos; RCF13 - Falta de mecanismos para monitorar e informar o principal sobre as atividades e desempenho do agente; CGT1 - Falta de materiais em consequência da eliminação dos estoques -pulmão; RCF2 - Oportunismo por parte de fornecedores e/ou clientes; RCF5 Baixa capacidade dos fornecedores em cumprir com as quantidades programa- 
das; DEM6 - Falha na rapidez de entrega (aumento nos tempos entre o recebimento do pedido e a entrega ao cliente); AIN7 - Conjunto de habilidades (competências) dos funcionários; AIN5 Falta de eficiência dos processos que permitem o fluxo de materiais, desde a matéria-prima até o produto final (ou usuário final); DEM4 - Falta de habilidade para lidar com mudança de produto na demanda; AEX2 - Globalização da concorrência; AIN15 - Falta de capacidade da empresa em processar informações e AIN12 - Condições da infraestrutura interna de TI.

\section{CONSIDERAÇÕES FINAIS}

O objetivo desta pesquisa era de identificar os diversos riscos que ocorrem em cadeias de suprimentos. Partindo de uma tipologia de riscos em cadeias de suprimentos, através de uma survey se investigou empiricamente quais os riscos que exigem maior atenção dos gestores das cadeias de suprimentos.

A análise dos dados indicou quais são esses riscos nas empresas e cadeias de suprimentos investigadas. Os riscos relativos à estrutura organizacional demonstram que existe preocupação com a demora na implementação de mudanças organizacionais mais rapidamente, bem como, com a complexidade estrutural das cadeias, fluxos de materiais e informações e, ainda com a dispersão geográfica de fornecedores e clientes, o que pode ser entendido como uma dependência dos sistemas de transportes. Esse resultado é compatível com o resultado encontrado para a falta de eficiência dos processos que permitem o fluxo de materiais, desde a matéria-prima até o produto final (ou usuário final). Não se esperava que a infraestrutura externa de TI e desastres naturais constituíssem riscos às cadeias de suprimento na região do Sul do Brasil. Entretanto, aparecem como risco a exigir atenção nas cadeias de suprimento. Muito possivelmente, esses gestores enfrentaram esse tipo de dificuldade em passado recente. Isso pode ser explicado através de um raciocínio de cunho psicológico amplamente aceito no que diz respeito ao julgamento equivocado do impacto de interrupções em cadeias de suprimentos. Uma pesquisa realizada por psicólogos mostrou que as pessoas, ao invés de usarem estatísticas, se valem de uma quantidade limitada de heurísticas para prever o impacto dos riscos (KAHNEMAN; TVERSKY, 1973). Uma delas é denominada "heurística da disponibilidade" (SLOVIC et al., 1982). Os seres humanos fazem julgamentos com base naquilo de que se lembram, ao invés de em dados completos. Esses indivíduos podem facilmente se lembrar de desastres naturais, ocorridos meses antes da pesquisa naquela região do Brasil. A falta de capacidade dos fornecedores em cumprir com os prazos de entrega e o desalinhamento estratégico (existência de conflito de objetivos) entre os membros da cadeia de suprimentos, o oportunismo por parte de fornecedores e/ou clientes, as falhas de fornecedores em gerar reduções de custos e a falta de mecanismos para monitorar e informar o principal sobre as atividades e desempenho, baixa capacidade dos fornecedores em cumprir com as quantidades programadas apontam na direção de que ainda as CS não atingiram de forma plena os objetivos relativos aos relacionamentos com fornecedores $\mathrm{e}$ clientes. A falha na rapidez de entrega (aumento nos tempos entre o recebimento do pedido e a entrega ao cliente), as informações insuficientes ou distorcidas dadas pelos clientes sobre quantidades de pedidos ou demanda e a falta de habilidade para lidar com mudança de produto na demanda representam riscos relacionados à demanda bastante citadas na literatura sobre riscos em CS. O ritmo inferior à média do setor, na geração de inovações em produtos e processos e a inabilidade de se manter atualizada em relação ao desenvolvimento tecnológico 
do setor demonstram compatibilidade dos respondentes e preocupação com a inovação. Da mesma forma, a falta de capacidade da empresa em processar informações e as condições da infraestrutura interna de TI, na percepção dos respondentes, representam preocupações com a TI e com o grande fluxo de informações ao qual as empresas ficam submetidas.

Os resultados qualificam o atual interesse no assunto, especialmente no que concerne aos riscos envolvidos com a estrutura organizacional, relacionamentos com fornecedores e clientes, demanda, ambiente externo e interno e infraestrutura e sugerem aos gestores que, antes de qualquer coisa, voltem suas atenções para esses riscos que são comprovadamente o "feijão com arroz" da gestão de cadeias de suprimentos.

Por fim, como sugestões de pesquisas futuras, devido as diversas reflexões e discussões dos/entre os autores, durante a elaboração desse artigo, relacionamos as seguintes:

1. posicionar a gestão de riscos em cadeias de suprimentos dentro de uma literatura mais ampla sobre teoria do risco e a prática da gestão de risco;

2. identificar os conceitos-chave de risco e gestão de risco vindo de outras disciplinas dentro do contexto de cadeia de suprimento;

3. desenvolver de um modelo que mapeie as cadeias de suprimentos tradicionais, enxutas, ágeis quanto às dimensões da sensibilidade ao cliente e competência de atenuação de riscos;

4. testar empiricamente as relações diretas entre determinadas variáveis de risco do contexto (teoria da contingência), a estrutura da gestão de riscos e o desempenho das cadeias de suprimentos;

5. identificar os fatores potencializadores da resiliência das Cadeias de suprimento;

6. buscar o entendimento das implicações da avaliação dos riscos entre nós das cadeias de suprimentos através da Economia dos Custos de Transação e Teoria da Agência;

7. verificar, medir se a estratégia de gestão dos riscos das cadeias de suprimentos atua como mediadora na associação entre a cadeia de suprimentos e o desempenho das cadeias de suprimentos.

\section{Referências}

AGUIAR, E. C. Contribuição ao estudo do fator risco no desempenho em organizações e cadeias de suprimentos. São Paulo, 2010. 177f. Tese (Doutorado em Administração .(Faculdade de Economia e Administração, USP, São Paulo, 2010.

ALBERTON, A. Uma Metodologia para auxiliar no gerenciamento de riscos e na seleção de alternativas de investimentos em segurança. Florianópolis, 199273 .6f. Dissertação ) Mestrado em Engenharia de Produção .( Universidade Federal de Santa Catarina. Florianópolis, .1996

BALLOU, R. H. Gerenciamento da Cadeia de Suprimentos/Logística Empresarial. 5a. Edicao. Porto Alegre, Ed.Bookman, 2006.

BASTIAS, H. H. Introducción a la ingeniería de prevención de pérdidas. São Paulo: Conselho Regional do Estado de São Paulo da Associação Brasileira para a Prevenção de Acidentes, 19 .97

BLACKHURST, J.; DUNN, K.S.; CRAIGHEAD, C.W. An Empirically 
IDENTIFICAÇÃO DOS RISCOS EM CADEIAS DE SUPRIMENTOS: um estudo introdutório com empresas da região Sul do Brasil.

Derived Framework of Global Supply Resiliency .Journal of Business Logistics, $.2011,391-374,(4) 32$

CHOPRA, S.; SODHI, M. S. Managing risk to avoid supply chain breakdown . MIT Sloan Management Review] .S.1.], v. 46, n. 1, 2005.

DANI, S; GHADGE, A. Supply chain risk and resilience: The past decade and future directions. In: Proceedings of papers of the 17th International Symposium on Logistics (ISL 2012). New Horizons in Logistics and Supply Chain Management. Nottingham University. Disponível em http://eprints.hud.ac.uk. Acesso em 30 set 2014.

DI SÉRIO L. C.; SAMPAIO, M. Projeto da Cadeia de Suprimentos: uma visão dinâmica da decisão de fazer versus comprar. RAE. v.41, n.1, p.54-66, jan./ mar. 2001.

FRANCESCHINI ,P. G.; GURGEL ,F. A. Administração de materiais e do patrimônio .São Paulo: Pioneira Thomson, 2002.

FLEURY ,A; FLEURY ,M. T. L. Estratégias empresariais e formação de competências .São Paulo: Atlas, 2000.

FLEURY ,P. F. Conceito de Logística Integrada e Supply Chain Management . In: FLEURY, P.F.; WANKE, P.; FIGUEIREDO, K.F. Logística Empresarial .São Paulo: Atlas, 2000.

FOLHA DE SÃO PAULO. Exército Ocupa Campos de Petróleo e Gás na Bolívia, Caderno Mundo, 01/05/2006.

FURLANETTO,E. L. Estruturação das cadeias de suprimentos e a decisão fazer versus comprar. In: XXII Simpósio de Gestão da Inovação Tecnológica. Salvador, 2002.
GIL, A. C .Como elaborar projetos de pesquisa .São Paulo: Atlas, 2002.

HAIR JR. ,Joseph F.; ANDERSON, Rolph E.; TATHAM, Ronald L.; BLACK, William C. Análise multivariada de dados .Tradução de Anselmo Chaves Neto. Porto Alegre: Bookman, 2005.

HALLIKAS,J.; KARVONEN, I.; PULKKINEN, U.; VIROLAINEN, V.M.; TUOMINEN, M. Risk management processes in supplier networks. International Journal of Production Economics, 90(1), 4758. 2004.

;.R ,BRENCHLEY ;.C ,HARLAND .H ,WALKERRisk in supply networks. Journal of Purchasing and Supply Management] .S.1.], 2003.

HULETT, D. T. Key Characteristics of a mature risk management process .New York, NY USA :International Institute for Learning Inc.2002,

JACKSON, N.; CARTER, P. The perception of risk .In.: ANSELL, Jake, WHARTON, Frank. Risk: analysis assessment and management .England: John Wiley \& Sons Ltda., 1992.

JÜTTNER, U. Supply chain risk management - Understanding the business requirements from a practitioner perspective. International Journal of Logistics Management,Vol.16, No 1. 2005.

;.H $\quad$,P E C K $\quad$;.U , J Ü T T N E R .M ,CHRISTOPHERSupply chain risk management outlining an agenda for future research .International Journal of Logistics: Research and Applications] . S.1.], v. 6, n. 5, 2003.

KAHNEMAN, D.; TVERSKY, A. On the psychology of prediction. Psychological Review] .S.1.],-237:(4)80 $.1973,251$ 
LUMMUS, R. VOKURKA, R.. Defining supply chain management: A historical perspective and practical guidelines. Industrial Management and Data Systems, 99, 11-17. 1999.

MARBLE, R.P.; THUN, J.H. The Theory of Constraints and the Constraints of Theory: A Structural Equation Model for Supply Chain Disruptions. 17th Annual Conference of Production and Operations Management Society, Boston, MA, April 28 - May 1, 2006.

MARCH, J. G.; SHAPIRA, Z. Managerial perspectives on risk and risk taking .Management Science] .S.1.], .1987, 1418-1404:(11)33

MOORE, P.G. The Business of Risk, Cambridge University Press, Cambridge. 1983 .

NORMANN, A; LINDROTH, R. Categorization of Supply Chain Risk and Risk Management. In: BRINDLEY, C. Supply Chain Risk. Hampshire: Ashgate. 2004.

NUTINI, M. A importância de controlar os riscos empresariais para o alcance dos objetivos -Embraer. Excelência Resenha da Fundação Nacional de Qualidade ,ano 5 ,n ,38 .abr.2005 .

PARRA, P. H.; PIRES, S. R.I. Análise da gestão da cadeia de suprimentos na indústria de computadores. Gestão e Produção] .S.1.], v. ,10 n. ,1 p. ,15-1 abr. 2003.

POIRIER, C. P; REITER, S. E. Otimizando sua rede de negócios .São Paulo: Futura, 1997.

PIRES, S. R. I. Gestão da cadeia de suprimentos :conceitos, estratégias, práticas e casos - supply chain management .São Paulo: Atlas, 2004.
$\mathrm{SHI}, \mathrm{D}$.A review of enterprise supply chain risk management .Journal of Systems Science and Systems Engineering] .S.1.], v. 13, n. 2, June 2004.

SILVA FILHO, J. M.; ALVAREZ, R. R.; PROENÇA, A. Redes simétricas e seu processo de definição de estratégia coletiva e modelo de governança: propostas no âmbito das redes de incubadoras e parques tecnológicos .In: XXII Simpósio de Gestão de Inovação Tecnológica. Salvador, 2002.

SINGHAL, P; AGARWAL, G; MITTAL, M. L. Supply chain risk management: review, classification and future research directions. International Journal of Business Science and Applied Management, Volume 6, 2012.

SLOVIC, P.; FISCHHOFF, B., LICHTENSTEIN, S. Facts versus fears: understanding perceived risk. In: KAHNEMAN, D.; SLOVIC, P.; TVERSKY, A. (eds.). Judgment under uncertainty: heuristics and biases . Cambridge: Cambridge University Press, p. 463-489, 1982.

SODHI, M.S.; GAK-SON, B; TANG, C. S. Researchers' Perspectives on Supply Chain Risk Management. Production and Operations Management. Vol. 21, No. 1, pp. 1-13, January-February. 2012.

SOUZA, D.; KLIEMANN NETO, F.; ANZANELLO, M.. Avaliação de desempenho da cadeia de suprimentos balizada por critérios de competitividade empresarial. Produção Online, v.12, n.3, p.756-778.2012.

SVENSSON, G. The Principle of Balance Between Companies Inventories and Disturbances in Logistics Flows. International Journal of Physical Distribution and Logistics Management 33(9): 765-84. 2003. 
IDENTIFICAÇÃO DOS RISCOS EM CADEIAS DE SUPRIMENTOS: um estudo introdutório com empresas da região Sul do Brasil.

TANG, O.; MUSA, S.. Identifying risk issues and research advancements in supply chain risk management. International Journal of Production Economics, v.133, n.1, p.25-34. 2011.

TOMAS R. N.; ALCANTARA, R. L. C.. Modelos para gestão de riscos em cadeias de suprimentos: revisão, análise e diretrizes para futuras pesquisas. Gestão da Produção, vol.20, no. 3 : São Carlos, 2013.

TOMASKOVIC-DEVEY, D.; LEITER, J.; THOMPSON, S. Organizational survey nonresponse. Administrative Science Quarterly] .S.1.], ,457-439:(3)39 .1994

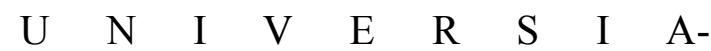
KNOWLEGE@WHARTON. Flexibilidade ante o desastre: gerenciando o risco de ruptura na cadeia de suprimentos .Disponível em: http://www. wharton.universia.net . Acesso em: 12 dez. 2009.

VILKO, J.; HALLIKAS, J.. Risk assessment in multimodal supply chains. International Journal of Production Economics, v.140, n.2, p.586-595. 2012.

VIVALDINI, M.; PIRES, S.; SOUZA, F.. Collaboration and Competition between 4PL and 3PL: $A$ study of a fastfood supply chain. Journal of Operations and Supply Chain Management. Brasil, 01 out. 2013. Disponível em http:// bibliotecadigital.fgv.br. Acesso: 26 set. 2014.

\section{.C ,BODE ;.M .S ,WAGNERAn} empirical investigation of supply chain performance along several dimensions of risk. Journal of Business Logistics] . S.1.], v. 29, n. 1, p. 307-325, 2008. WOOD JR., T. ZUFFO, P. K. Supply chain management .Revista de
Administração de Empresas] .S.1.], v. 38, n. 3, jul/.set.1998 .

ZSIDISIN, G. A.; MELNYK, S. A.; RAGATZ, G.L. An institutional theory perspective of business continuity planning for purchasing and supply management .International Journal of Production Research] .S.1.], 08/2005. 